\title{
Neurosteroid Analogues. 11. Alternative Ring System Scaffolds: GABA Modulatory and Anesthetic Actions of Benz[f]indenes
}

Jamie B. Scaglione, Brad D. Manion, Ann Benz, Amanda Taylor, Gregory T. DeKoster, Nigam P. Rath, Alex S. Evers, Charles F. Zorumski, Steven Mennerick, and Douglas F. Covey

Supporting Information

Table of Contents

Elemental Analyses for Compounds 1a, 1b, 1c, 1d, 2a, 2b, 2c, 2d, 3a, and 3b...........S2

X-ray Crystallographic Data for Compound 8a................................S3-S12

Projection View of Compound 8a......................................................

X-ray Crystallographic Data for Compound 1c...............................S14-S23

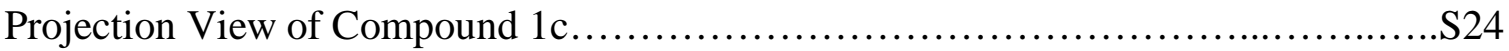


Table 1. Elemental Analyses for Target Compounds

\begin{tabular}{cccccccc} 
& & \multicolumn{3}{c}{ Calculated } & \multicolumn{3}{c}{ Found } \\
Compound & Formula & $\mathrm{C}$ & $\mathrm{H}$ & $\mathrm{N}$ & $\mathrm{C}$ & $\mathrm{H}$ & $\mathrm{N}$ \\
$\mathbf{1 a}$ & $\mathrm{C}_{16} \mathrm{H}_{26} \mathrm{O}_{2}$ & 76.75 & 10.47 & - & 76.95 & 10.27 & - \\
$\mathbf{1 b}$ & $\mathrm{C}_{16} \mathrm{H}_{26} \mathrm{O}_{2}$ & 76.75 & 10.47 & - & 76.55 & 10.48 & - \\
$\mathbf{1 c}$ & $\mathrm{C}_{16} \mathrm{H}_{26} \mathrm{O}_{2}$ & 76.75 & 10.47 & - & 76.89 & 10.37 & - \\
$\mathbf{1 d}$ & $\mathrm{C}_{16} \mathrm{H}_{26} \mathrm{O}_{2}$ & 76.75 & 10.47 & - & 76.56 & 10.22 & - \\
$\mathbf{2 a}$ & $\mathrm{C}_{17} \mathrm{H}_{27} \mathrm{NO}$ & 78.11 & 10.41 & 5.36 & 77.94 & 10.39 & 5.11 \\
$\mathbf{2 b}$ & $\mathrm{C}_{17} \mathrm{H}_{27} \mathrm{NO}$ & 78.11 & 10.41 & 5.36 & 78.26 & 10.58 & 5.43 \\
$\mathbf{2 c}$ & $\mathrm{C}_{17} \mathrm{H}_{27} \mathrm{NO}$ & 78.11 & 10.41 & 5.36 & 78.38 & 10.19 & 5.26 \\
$\mathbf{2 d}$ & $\mathrm{C}_{17} \mathrm{H}_{27} \mathrm{NO}$ & 78.11 & 10.41 & 5.36 & 78.17 & 10.19 & 5.24 \\
3a & $\mathrm{C}_{18} \mathrm{H}_{30} \mathrm{O}_{2}$ & 77.65 & 10.86 & - & 77.70 & 11.09 & - \\
3b & $\mathrm{C}_{18} \mathrm{H}_{30} \mathrm{O}_{2}$ & 77.65 & 10.86 & - & 77.49 & 11.00 & -
\end{tabular}


Table 2. Crystal data and structure refinement for $8 \mathrm{a}$.

Name of the Compound $[1 S, 3 \mathrm{a} S, 4 \mathrm{a} S, 6 S, 8 \mathrm{a} R, 9 \mathrm{a} S]$-Dodecahydro-1-hydroxy-9a-methyl-1 $H$-benz $[f$ indene6-ethanol

Empirical formula

Formula weight

Temperature

Wavelength

Crystal system

Space group

Unit cell dimensions

Volume

Z

Density (calculated)

Absorption coefficient

$\mathrm{F}(000)$

Crystal size

Theta range for data collection

Index ranges

Reflections collected

Independent reflections

Completeness to theta $=25.00^{\circ}$

Absorption correction

Max. and min. transmission

Refinement method

Data / restraints / parameters

Goodness-of-fit on $\mathrm{F}^{2}$

Final $\mathrm{R}$ indices [I $>2 \operatorname{sigma}(\mathrm{I})]$

$\mathrm{R}$ indices (all data)

Absolute structure parameter

Extinction coefficient

Largest diff. peak and hole
$\mathrm{C}_{16} \mathrm{H}_{28} \mathrm{O}_{2}$

252.38

295(2) K

$0.71073 \AA$

Orthorhombic

$\mathrm{P} 2{ }_{1} 2_{1} 2_{1}$

$\mathrm{a}=10.8574(3) \AA$

$\alpha=90^{\circ}$.

$\mathrm{b}=11.4080(3) \AA$

$\beta=90^{\circ}$.

$\mathrm{c}=24.5297(8) \AA$ $\gamma=90^{\circ}$.

3038.28(15) $\AA^{3}$

8

$1.104 \mathrm{Mg} / \mathrm{m}^{3}$

$0.070 \mathrm{~mm}^{-1}$

1120

$0.36 \times 0.34 \times 0.30 \mathrm{~mm}^{3}$

1.97 to $25.00^{\circ}$.

$-12 \leq \mathrm{h} \leq 2,-13 \leq \mathrm{k} \leq 3,-29 \leq \mathrm{l} \leq 29$

27056

$5344[\mathrm{R}(\mathrm{int})=0.04$

$100.0 \%$

Semi-empirical from equivalents

0.9793 and 0.9752

Full-matrix least-squares on $\mathrm{F}^{2}$

5344 / 1 / 335

1.055

$\mathrm{R} 1=0.0554, \mathrm{wR} 2=0.1596$

$\mathrm{R} 1=0.0922, \mathrm{wR} 2=0.1826$

$-1(2)$

$0.0104(19)$

0.170 and -0.144 e. $\AA^{-3}$ 
Table 3. Atomic coordinates ( $\mathrm{x} 10^{4}$ ) and equivalent isotropic displacement parameters $\left(\AA^{2} \times 10^{3}\right)$ for $8 \mathrm{a}$. $U(\mathrm{eq})$ is defined as one third of the trace of the orthogonalized $\mathrm{U}^{\mathrm{ij}}$ tensor.

\begin{tabular}{|c|c|c|c|c|}
\hline & $\mathrm{x}$ & $\mathrm{y}$ & $\mathrm{z}$ & $\mathrm{U}(\mathrm{eq})$ \\
\hline $\mathrm{O}\left(1^{\prime}\right)$ & $3309(2)$ & $8821(2)$ & $-1769(1)$ & $95(1)$ \\
\hline $\mathrm{O}\left(3^{\prime}\right)$ & $4308(3)$ & $8320(2)$ & $7232(1)$ & $125(1)$ \\
\hline $\mathrm{O}\left(1^{\prime} \mathrm{A}\right)$ & $7403(5)$ & $8306(5)$ & 2867(2) & $115(2)$ \\
\hline $\mathrm{O}\left(1^{\prime} \mathrm{B}\right)$ & $6795(7)$ & 7381(5) & $3137(3)$ & $135(2)$ \\
\hline $\mathrm{O}(3)$ & $6065(3)$ & $6855(3)$ & 2134(1) & $136(1)$ \\
\hline $\mathrm{C}(1)$ & $2802(4)$ & 7532(4) & 1933(2) & $126(2)$ \\
\hline$C(2)$ & $3834(4)$ & 6892(5) & $2248(2)$ & $129(2)$ \\
\hline $\mathrm{C}(3)$ & 4949(4) & 6837(4) & 1851(1) & $107(1)$ \\
\hline$C(4)$ & $5345(3)$ & 7697(3) & 891(1) & $80(1)$ \\
\hline$C(5)$ & 5412(3) & $8320(3)$ & $-99(1)$ & $82(1)$ \\
\hline$C(6)$ & $4917(3)$ & 9148(3) & $-528(1)$ & $84(1)$ \\
\hline $\mathrm{C}(7)$ & $3526(3)$ & $9121(2)$ & $-581(1)$ & $71(1)$ \\
\hline $\mathrm{C}(8)$ & 2943(3) & 9271(3) & $-17(1)$ & $76(1)$ \\
\hline$C(9)$ & $2769(3)$ & $8523(3)$ & $954(1)$ & $87(1)$ \\
\hline$C(3 \mathrm{~A})$ & $4708(3)$ & 7832(3) & 1439(1) & $85(1)$ \\
\hline $\mathrm{C}(4 \mathrm{~A})$ & $4831(2)$ & $8510(3)$ & $459(1)$ & $72(1)$ \\
\hline $\mathrm{C}(8 \mathrm{~A})$ & $3423(2)$ & $8428(3)$ & $405(1)$ & $68(1)$ \\
\hline$C(9 A)$ & $3322(3)$ & 7665(3) & $1350(1)$ & $88(1)$ \\
\hline $\mathrm{C}\left(3 \mathrm{~A}^{\prime}\right)$ & $5012(3)$ & $9022(4)$ & 1694(2) & $112(1)$ \\
\hline$C\left(2^{\prime}\right)$ & 2999(3) & 9993(3) & $-980(1)$ & $87(1)$ \\
\hline $\mathrm{C}\left(1^{\prime}\right)$ & $3496(4)$ & 9940(3) & $-1553(1)$ & 91(1) \\
\hline $\mathrm{C}\left(1^{\prime} 2\right)$ & $6033(4)$ & 10821(3) & $6711(2)$ & $115(1)$ \\
\hline$C\left(2^{\prime} 2\right)$ & $5215(4)$ & $10256(3)$ & $7146(1)$ & $100(1)$ \\
\hline$C\left(3^{\prime} 2\right)$ & $4543(3)$ & $9260(3)$ & $6859(1)$ & $89(1)$ \\
\hline$C\left(4^{\prime} 2\right)$ & $4765(3)$ & $8297(3)$ & $5904(1)$ & $92(1)$ \\
\hline$C\left(5^{\prime} 2\right)$ & 4901(4) & $7729(3)$ & $4909(1)$ & $111(1)$ \\
\hline $\mathrm{C}\left(6^{\prime} 2\right)$ & $5695(4)$ & 7712(4) & $4400(2)$ & $114(1)$ \\
\hline$C\left(7^{\prime} 2\right)$ & $6123(3)$ & 8896(3) & $4241(1)$ & $90(1)$ \\
\hline $\mathrm{C}\left(8^{\prime} 2\right)$ & $6724(3)$ & 9512(3) & $4723(1)$ & $90(1)$ \\
\hline $\mathrm{C}\left(9^{\prime} 2\right)$ & $6535(3)$ & $10202(3)$ & $5699(1)$ & $85(1)$ \\
\hline $\mathrm{C}(3 \mathrm{~A} 2)$ & $5359(3)$ & $8938(2)$ & 6374(1) & $74(1)$ \\
\hline
\end{tabular}




\begin{tabular}{lrrrr}
$\mathrm{C}(3 \mathrm{~B} 2)$ & $6514(4)$ & $8271(3)$ & $6582(2)$ & $110(1)$ \\
$\mathrm{C}(4 \mathrm{~A} 2)$ & $5521(3)$ & $8308(3)$ & $5386(1)$ & $80(1)$ \\
$\mathrm{C}(8 \mathrm{~A} 2)$ & $5925(3)$ & $9542(3)$ & $5229(1)$ & $73(1)$ \\
$\mathrm{C}(9 \mathrm{~A} 2)$ & $5697(3)$ & $10181(2)$ & $6186(1)$ & $77(1)$ \\
$\mathrm{C}\left(1^{\prime} \mathrm{B}\right)$ & $6510(5)$ & $8418(6)$ & $3245(2)$ & $160(2)$ \\
$\mathrm{C}\left(2{ }^{\prime} \mathrm{B}\right)$ & $6992(4)$ & $8907(4)$ & $3753(2)$ & $118(1)$ \\
& & & & \\
\hline
\end{tabular}


Table 4. Bond lengths $[\AA]$ and angles $\left[{ }^{\circ}\right]$ for $8 \mathrm{a}$.

\begin{tabular}{|c|c|}
\hline $\mathrm{O}\left(1^{\prime}\right)-\mathrm{C}\left(1^{\prime}\right)$ & $1.397(4)$ \\
\hline $\mathrm{O}\left(3^{\prime}\right)-\mathrm{C}\left(3^{\prime} 2\right)$ & $1.433(4)$ \\
\hline $\mathrm{O}\left(1^{\prime} \mathrm{A}\right)-\mathrm{C}\left(1^{\prime} \mathrm{B}\right)$ & $1.347(7)$ \\
\hline $\mathrm{O}\left(1^{\prime} \mathrm{B}\right)-\mathrm{C}\left(1^{\prime} \mathrm{B}\right)$ & $1.251(7)$ \\
\hline $\mathrm{O}(3)-\mathrm{C}(3)$ & $1.397(5)$ \\
\hline $\mathrm{C}(1)-\mathrm{C}(9 \mathrm{~A})$ & $1.545(4)$ \\
\hline $\mathrm{C}(1)-\mathrm{C}(2)$ & $1.544(6)$ \\
\hline$C(2)-C(3)$ & $1.555(5)$ \\
\hline $\mathrm{C}(3)-\mathrm{C}(3 \mathrm{~A})$ & $1.542(5)$ \\
\hline $\mathrm{C}(4)-\mathrm{C}(4 \mathrm{~A})$ & $1.514(4)$ \\
\hline $\mathrm{C}(4)-\mathrm{C}(3 \mathrm{~A})$ & $1.519(4)$ \\
\hline$C(5)-C(6)$ & $1.513(4)$ \\
\hline $\mathrm{C}(5)-\mathrm{C}(4 \mathrm{~A})$ & $1.524(4)$ \\
\hline $\mathrm{C}(6)-\mathrm{C}(7)$ & $1.515(4)$ \\
\hline $\mathrm{C}(7)-\mathrm{C}\left(2^{\prime}\right)$ & $1.508(4)$ \\
\hline$C(7)-C(8)$ & $1.530(4)$ \\
\hline $\mathrm{C}(8)-\mathrm{C}(8 \mathrm{~A})$ & $1.506(4)$ \\
\hline $\mathrm{C}(9)-\mathrm{C}(9 \mathrm{~A})$ & $1.505(4)$ \\
\hline $\mathrm{C}(9)-\mathrm{C}(8 \mathrm{~A})$ & $1.526(4)$ \\
\hline $\mathrm{C}(3 \mathrm{~A})-\mathrm{C}\left(3 \mathrm{~A}^{\prime}\right)$ & $1.531(5)$ \\
\hline $\mathrm{C}(3 \mathrm{~A})-\mathrm{C}(9 \mathrm{~A})$ & $1.533(5)$ \\
\hline $\mathrm{C}(4 \mathrm{~A})-\mathrm{C}(8 \mathrm{~A})$ & $1.537(4)$ \\
\hline$C\left(2^{\prime}\right)-C\left(1^{\prime}\right)$ & $1.508(5)$ \\
\hline $\mathrm{C}\left(1^{\prime} 2\right)-\mathrm{C}(9 \mathrm{~A} 2)$ & $1.525(5)$ \\
\hline $\mathrm{C}\left(1^{\prime} 2\right)-\mathrm{C}\left(2^{\prime} 2\right)$ & $1.531(5)$ \\
\hline$C\left(2^{\prime} 2\right)-C\left(3^{\prime} 2\right)$ & $1.523(5)$ \\
\hline$C\left(3^{\prime} 2\right)-C(3 A 2)$ & $1.527(4)$ \\
\hline $\mathrm{C}\left(4^{\prime} 2\right)-\mathrm{C}(3 \mathrm{~A} 2)$ & $1.511(4)$ \\
\hline$C\left(4^{\prime} 2\right)-C(4 A 2)$ & $1.514(4)$ \\
\hline$C\left(5^{\prime} 2\right)-C(4 A 2)$ & $1.502(5)$ \\
\hline $\mathrm{C}\left(5^{\prime} 2\right)-\mathrm{C}\left(6^{\prime} 2\right)$ & $1.518(5)$ \\
\hline$C\left(6^{\prime} 2\right)-C\left(7^{\prime} 2\right)$ & $1.481(5)$ \\
\hline $\mathrm{C}\left(7^{\prime} 2\right)-\mathrm{C}\left(2^{\prime} \mathrm{B}\right)$ & $1.524(5)$ \\
\hline $\mathrm{C}\left(7^{\prime} 2\right)-\mathrm{C}\left(8^{\prime} 2\right)$ & $1.523(5)$ \\
\hline
\end{tabular}




\begin{tabular}{|c|c|}
\hline $\mathrm{C}\left(8^{\prime} 2\right)-\mathrm{C}(8 \mathrm{~A} 2)$ & $1.515(4)$ \\
\hline $\mathrm{C}\left(9^{\prime} 2\right)-\mathrm{C}(9 \mathrm{~A} 2)$ & $1.503(4)$ \\
\hline $\mathrm{C}\left(9^{\prime} 2\right)-\mathrm{C}(8 \mathrm{~A} 2)$ & $1.527(4)$ \\
\hline $\mathrm{C}(3 \mathrm{~A} 2)-\mathrm{C}(9 \mathrm{~A} 2)$ & $1.535(4)$ \\
\hline $\mathrm{C}(3 \mathrm{~A} 2)-\mathrm{C}(3 \mathrm{~B} 2)$ & $1.552(5)$ \\
\hline $\mathrm{C}(4 \mathrm{~A} 2)-\mathrm{C}(8 \mathrm{~A} 2)$ & $1.523(4)$ \\
\hline $\mathrm{C}\left(1^{\prime} \mathrm{B}\right)-\mathrm{C}\left(2^{\prime} \mathrm{B}\right)$ & $1.462(6)$ \\
\hline $\mathrm{C}(9 \mathrm{~A})-\mathrm{C}(1)-\mathrm{C}(2)$ & 104.1(3) \\
\hline$C(1)-C(2)-C(3)$ & $105.7(3)$ \\
\hline $\mathrm{O}(3)-\mathrm{C}(3)-\mathrm{C}(3 \mathrm{~A})$ & $117.6(4)$ \\
\hline $\mathrm{O}(3)-\mathrm{C}(3)-\mathrm{C}(2)$ & $111.3(3)$ \\
\hline $\mathrm{C}(3 \mathrm{~A})-\mathrm{C}(3)-\mathrm{C}(2)$ & $104.4(3)$ \\
\hline $\mathrm{C}(4 \mathrm{~A})-\mathrm{C}(4)-\mathrm{C}(3 \mathrm{~A})$ & $112.9(2)$ \\
\hline$C(6)-C(5)-C(4 A)$ & $112.9(2)$ \\
\hline$C(5)-C(6)-C(7)$ & $113.6(2)$ \\
\hline$C\left(2^{\prime}\right)-C(7)-C(6)$ & $114.8(3)$ \\
\hline $\mathrm{C}\left(2^{\prime}\right)-\mathrm{C}(7)-\mathrm{C}(8)$ & $110.8(2)$ \\
\hline$C(6)-C(7)-C(8)$ & $109.5(2)$ \\
\hline $\mathrm{C}(8 \mathrm{~A})-\mathrm{C}(8)-\mathrm{C}(7)$ & $113.9(2)$ \\
\hline $\mathrm{C}(9 \mathrm{~A})-\mathrm{C}(9)-\mathrm{C}(8 \mathrm{~A})$ & $109.7(2)$ \\
\hline$C(4)-C(3 A)-C\left(3 A^{\prime}\right)$ & $110.7(3)$ \\
\hline $\mathrm{C}(4)-\mathrm{C}(3 \mathrm{~A})-\mathrm{C}(9 \mathrm{~A})$ & $108.0(2)$ \\
\hline$C\left(3 A^{\prime}\right)-C(3 A)-C(9 A)$ & $112.3(3)$ \\
\hline $\mathrm{C}(4)-\mathrm{C}(3 \mathrm{~A})-\mathrm{C}(3)$ & $115.4(3)$ \\
\hline$C\left(3 A^{\prime}\right)-C(3 A)-C(3)$ & $110.3(3)$ \\
\hline $\mathrm{C}(9 \mathrm{~A})-\mathrm{C}(3 \mathrm{~A})-\mathrm{C}(3)$ & $99.7(3)$ \\
\hline $\mathrm{C}(4)-\mathrm{C}(4 \mathrm{~A})-\mathrm{C}(5)$ & $112.9(2)$ \\
\hline $\mathrm{C}(4)-\mathrm{C}(4 \mathrm{~A})-\mathrm{C}(8 \mathrm{~A})$ & $113.0(2)$ \\
\hline $\mathrm{C}(5)-\mathrm{C}(4 \mathrm{~A})-\mathrm{C}(8 \mathrm{~A})$ & 109.0(2) \\
\hline $\mathrm{C}(8)-\mathrm{C}(8 \mathrm{~A})-\mathrm{C}(9)$ & $113.5(2)$ \\
\hline $\mathrm{C}(8)-\mathrm{C}(8 \mathrm{~A})-\mathrm{C}(4 \mathrm{~A})$ & $111.4(2)$ \\
\hline $\mathrm{C}(9)-\mathrm{C}(8 \mathrm{~A})-\mathrm{C}(4 \mathrm{~A})$ & $112.4(2)$ \\
\hline $\mathrm{C}(9)-\mathrm{C}(9 \mathrm{~A})-\mathrm{C}(3 \mathrm{~A})$ & $113.7(3)$ \\
\hline $\mathrm{C}(9)-\mathrm{C}(9 \mathrm{~A})-\mathrm{C}(1)$ & 121.1(3) \\
\hline $\mathrm{C}(3 \mathrm{~A})-\mathrm{C}(9 \mathrm{~A})-\mathrm{C}(1)$ & $103.9(3)$ \\
\hline
\end{tabular}




\begin{tabular}{|c|c|}
\hline$C\left(1^{\prime}\right)-C\left(2^{\prime}\right)-C(7)$ & $116.3(3)$ \\
\hline $\mathrm{O}\left(1^{\prime}\right)-\mathrm{C}\left(1^{\prime}\right)-\mathrm{C}\left(2^{\prime}\right)$ & $109.7(3)$ \\
\hline $\mathrm{C}(9 \mathrm{~A} 2)-\mathrm{C}\left(1^{\prime} 2\right)-\mathrm{C}\left(2^{\prime} 2\right)$ & $104.4(3)$ \\
\hline $\mathrm{C}\left(3^{\prime} 2\right)-\mathrm{C}\left(2^{\prime} 2\right)-\mathrm{C}\left(1^{\prime} 2\right)$ & $105.6(3)$ \\
\hline $\mathrm{O}\left(3^{\prime}\right)-\mathrm{C}\left(3^{\prime} 2\right)-\mathrm{C}\left(2^{\prime} 2\right)$ & $110.3(3)$ \\
\hline $\mathrm{O}\left(3^{\prime}\right)-\mathrm{C}\left(3^{\prime} 2\right)-\mathrm{C}(3 \mathrm{~A} 2)$ & $114.8(3)$ \\
\hline$C\left(2^{\prime} 2\right)-C\left(3^{\prime} 2\right)-C(3 A 2)$ & $105.2(3)$ \\
\hline $\mathrm{C}(3 \mathrm{~A} 2)-\mathrm{C}\left(4^{\prime} 2\right)-\mathrm{C}(4 \mathrm{~A} 2)$ & $113.9(3)$ \\
\hline $\mathrm{C}(4 \mathrm{~A} 2)-\mathrm{C}\left(5^{\prime} 2\right)-\mathrm{C}\left(6^{\prime} 2\right)$ & $113.0(3)$ \\
\hline $\mathrm{C}\left(7^{\prime} 2\right)-\mathrm{C}\left(6^{\prime} 2\right)-\mathrm{C}\left(5^{\prime} 2\right)$ & $112.6(3)$ \\
\hline $\mathrm{C}\left(6^{\prime} 2\right)-\mathrm{C}\left(7^{\prime} 2\right)-\mathrm{C}\left(2^{\prime} \mathrm{B}\right)$ & $114.1(3)$ \\
\hline $\mathrm{C}\left(6^{\prime} 2\right)-\mathrm{C}\left(7^{\prime} 2\right)-\mathrm{C}\left(8^{\prime} 2\right)$ & $110.5(3)$ \\
\hline $\mathrm{C}\left(2^{\prime} \mathrm{B}\right)-\mathrm{C}\left(7^{\prime} 2\right)-\mathrm{C}\left(8^{\prime} 2\right)$ & $109.9(3)$ \\
\hline $\mathrm{C}(8 \mathrm{~A} 2)-\mathrm{C}\left(8^{\prime} 2\right)-\mathrm{C}\left(7^{\prime} 2\right)$ & $113.7(3)$ \\
\hline $\mathrm{C}(9 \mathrm{~A} 2)-\mathrm{C}\left(9^{\prime} 2\right)-\mathrm{C}(8 \mathrm{~A} 2)$ & 109.2(2) \\
\hline $\mathrm{C}\left(4^{\prime} 2\right)-\mathrm{C}(3 \mathrm{~A} 2)-\mathrm{C}\left(3^{\prime} 2\right)$ & $117.5(3)$ \\
\hline $\mathrm{C}\left(4^{\prime} 2\right)-\mathrm{C}(3 \mathrm{~A} 2)-\mathrm{C}(9 \mathrm{~A} 2)$ & $108.6(2)$ \\
\hline$C\left(3^{\prime} 2\right)-C(3 A 2)-C(9 A 2)$ & $98.6(2)$ \\
\hline $\mathrm{C}\left(4^{\prime} 2\right)-\mathrm{C}(3 \mathrm{~A} 2)-\mathrm{C}(3 \mathrm{~B} 2)$ & $111.0(3)$ \\
\hline $\mathrm{C}\left(3^{\prime} 2\right)-\mathrm{C}(3 \mathrm{~A} 2)-\mathrm{C}(3 \mathrm{~B} 2)$ & $109.3(3)$ \\
\hline $\mathrm{C}(9 \mathrm{~A} 2)-\mathrm{C}(3 \mathrm{~A} 2)-\mathrm{C}(3 \mathrm{~B} 2)$ & 111.1(3) \\
\hline $\mathrm{C}\left(5^{\prime} 2\right)-\mathrm{C}(4 \mathrm{~A} 2)-\mathrm{C}\left(4^{\prime} 2\right)$ & $114.0(3)$ \\
\hline $\mathrm{C}\left(5^{\prime} 2\right)-\mathrm{C}(4 \mathrm{~A} 2)-\mathrm{C}(8 \mathrm{~A} 2)$ & $109.8(2)$ \\
\hline$C\left(4^{\prime} 2\right)-C(4 A 2)-C(8 A 2)$ & $112.0(2)$ \\
\hline $\mathrm{C}\left(8^{\prime} 2\right)-\mathrm{C}(8 \mathrm{~A} 2)-\mathrm{C}(4 \mathrm{~A} 2)$ & $110.5(3)$ \\
\hline $\mathrm{C}\left(8^{\prime} 2\right)-\mathrm{C}(8 \mathrm{~A} 2)-\mathrm{C}\left(9^{\prime} 2\right)$ & $112.4(2)$ \\
\hline $\mathrm{C}(4 \mathrm{~A} 2)-\mathrm{C}(8 \mathrm{~A} 2)-\mathrm{C}\left(9^{\prime} 2\right)$ & $113.0(2)$ \\
\hline $\mathrm{C}\left(9^{\prime} 2\right)-\mathrm{C}(9 \mathrm{~A} 2)-\mathrm{C}\left(1^{\prime} 2\right)$ & $121.3(3)$ \\
\hline $\mathrm{C}\left(9^{\prime} 2\right)-\mathrm{C}(9 \mathrm{~A} 2)-\mathrm{C}(3 \mathrm{~A} 2)$ & $113.5(3)$ \\
\hline $\mathrm{C}\left(1^{\prime} 2\right)-\mathrm{C}(9 \mathrm{~A} 2)-\mathrm{C}(3 \mathrm{~A} 2)$ & $104.2(2)$ \\
\hline $\mathrm{O}\left(1^{\prime} \mathrm{B}\right)-\mathrm{C}\left(1^{\prime} \mathrm{B}\right)-\mathrm{O}\left(1^{\prime} \mathrm{A}\right)$ & $65.6(4)$ \\
\hline $\mathrm{O}\left(1^{\prime} \mathrm{B}\right)-\mathrm{C}\left(1^{\prime} \mathrm{B}\right)-\mathrm{C}\left(2^{\prime} \mathrm{B}\right)$ & $116.8(6)$ \\
\hline $\mathrm{O}\left(1^{\prime} \mathrm{A}\right)-\mathrm{C}\left(1^{\prime} \mathrm{B}\right)-\mathrm{C}\left(2^{\prime} \mathrm{B}\right)$ & $111.4(5)$ \\
\hline $\mathrm{C}\left(1^{\prime} \mathrm{B}\right)-\mathrm{C}\left(2^{\prime} \mathrm{B}\right)-\mathrm{C}\left(7^{\prime} 2\right)$ & $116.4(4)$ \\
\hline
\end{tabular}


Table 5. Anisotropic displacement parameters $\left(\AA^{2} \times 10^{3}\right)$ for 8a. The anisotropic displacement factor exponent takes the form: $-2 \pi^{2}\left[h^{2} a^{* 2} U^{11}+\ldots+2 h k a^{*} b^{*} U^{12}\right]$

\begin{tabular}{|c|c|c|c|c|c|c|}
\hline & $\mathrm{U}^{11}$ & $\mathrm{U}^{22}$ & $\mathrm{U}^{33}$ & $\mathrm{U}^{23}$ & $\mathrm{U}^{13}$ & $\mathrm{U}^{12}$ \\
\hline $\mathrm{O}\left(1^{\prime}\right)$ & $109(2)$ & $82(1)$ & $95(1)$ & $-8(1)$ & $0(1)$ & $-15(1)$ \\
\hline $\mathrm{O}\left(3^{\prime}\right)$ & 171(3) & $113(2)$ & $91(2)$ & $3(1)$ & $29(2)$ & $-51(2)$ \\
\hline $\mathrm{O}\left(1^{\prime} \mathrm{A}\right)$ & $170(5)$ & $113(4)$ & $62(2)$ & $0(3)$ & $39(3)$ & $46(4)$ \\
\hline $\mathrm{O}\left(1^{\prime} \mathrm{B}\right)$ & $166(6)$ & $134(5)$ & $105(4)$ & $-39(4)$ & $-23(4)$ & $52(4)$ \\
\hline $\mathrm{O}(3)$ & $118(2)$ & $208(3)$ & $83(1)$ & $-13(2)$ & $-18(2)$ & $50(2)$ \\
\hline $\mathrm{C}(1)$ & $86(2)$ & $199(5)$ & $94(2)$ & 13(3) & $19(2)$ & $14(3)$ \\
\hline$C(2)$ & $124(3)$ & $177(4)$ & $84(2)$ & $10(3)$ & $23(2)$ & $24(3)$ \\
\hline$C(3)$ & $98(2)$ & $142(3)$ & $80(2)$ & $3(2)$ & $1(2)$ & $23(2)$ \\
\hline$C(4)$ & $57(2)$ & $97(2)$ & $87(2)$ & $-10(2)$ & $4(2)$ & $6(2)$ \\
\hline$C(5)$ & $55(2)$ & $97(2)$ & $93(2)$ & $-9(2)$ & $9(2)$ & $-5(2)$ \\
\hline$C(6)$ & $78(2)$ & $89(2)$ & $85(2)$ & $-7(2)$ & $14(2)$ & $-13(2)$ \\
\hline$C(7)$ & $71(2)$ & $58(2)$ & $85(2)$ & $-7(1)$ & $2(2)$ & $-8(1)$ \\
\hline $\mathrm{C}(8)$ & $62(2)$ & $80(2)$ & $86(2)$ & $-12(2)$ & $2(2)$ & $2(1)$ \\
\hline $\mathrm{C}(9)$ & $56(2)$ & $123(3)$ & $83(2)$ & $-11(2)$ & $9(2)$ & $9(2)$ \\
\hline $\mathrm{C}(3 \mathrm{~A})$ & $67(2)$ & $115(2)$ & $72(2)$ & $-16(2)$ & $2(2)$ & $13(2)$ \\
\hline $\mathrm{C}(4 \mathrm{~A})$ & $55(2)$ & $79(2)$ & $80(2)$ & $-17(2)$ & $5(1)$ & $-4(1)$ \\
\hline $\mathrm{C}(8 \mathrm{~A})$ & $54(2)$ & $75(2)$ & $76(2)$ & $-13(2)$ & $5(1)$ & $-2(1)$ \\
\hline $\mathrm{C}(9 \mathrm{~A})$ & $68(2)$ & $117(2)$ & $80(2)$ & $-5(2)$ & $12(2)$ & $3(2)$ \\
\hline$C\left(3 A^{\prime}\right)$ & $93(2)$ & $144(3)$ & $99(2)$ & $-41(2)$ & $-6(2)$ & $9(2)$ \\
\hline$C\left(2^{\prime}\right)$ & $104(2)$ & $67(2)$ & $90(2)$ & $-7(2)$ & $-3(2)$ & $3(2)$ \\
\hline $\mathrm{C}\left(1^{\prime}\right)$ & $119(3)$ & $64(2)$ & $91(2)$ & $-5(2)$ & $-5(2)$ & $-12(2)$ \\
\hline $\mathrm{C}\left(1^{\prime} 2\right)$ & $149(3)$ & $82(2)$ & $113(3)$ & $-16(2)$ & $11(3)$ & $-25(2)$ \\
\hline $\mathrm{C}\left(2^{\prime} 2\right)$ & $121(3)$ & $85(2)$ & $94(2)$ & $-2(2)$ & $18(2)$ & $2(2)$ \\
\hline$C\left(3^{\prime} 2\right)$ & $87(2)$ & $87(2)$ & $92(2)$ & $4(2)$ & $8(2)$ & $-8(2)$ \\
\hline $\mathrm{C}\left(4^{\prime} 2\right)$ & $104(2)$ & $81(2)$ & $91(2)$ & $8(2)$ & $4(2)$ & $-27(2)$ \\
\hline$C\left(5^{\prime} 2\right)$ & $152(4)$ & $84(2)$ & $97(2)$ & $2(2)$ & $-8(3)$ & $-24(2)$ \\
\hline $\mathrm{C}\left(6^{\prime} 2\right)$ & $148(3)$ & $96(3)$ & $98(3)$ & $-19(2)$ & $-21(3)$ & $12(2)$ \\
\hline$C\left(7^{\prime} 2\right)$ & $88(2)$ & $90(2)$ & $91(2)$ & $0(2)$ & $6(2)$ & $21(2)$ \\
\hline $\mathrm{C}\left(8^{\prime} 2\right)$ & $84(2)$ & $97(2)$ & $88(2)$ & $3(2)$ & $7(2)$ & $1(2)$ \\
\hline $\mathrm{C}\left(9^{\prime} 2\right)$ & $85(2)$ & $78(2)$ & $92(2)$ & $3(2)$ & $7(2)$ & $-16(2)$ \\
\hline $\mathrm{C}(3 \mathrm{~A} 2)$ & $79(2)$ & $60(2)$ & $82(2)$ & $3(1)$ & $4(2)$ & $-3(1)$ \\
\hline
\end{tabular}




\begin{tabular}{lcccccc}
$\mathrm{C}(3 \mathrm{~B} 2)$ & $134(3)$ & $96(2)$ & $102(2)$ & $10(2)$ & $-15(2)$ & $35(2)$ \\
$\mathrm{C}(4 \mathrm{~A} 2)$ & $94(2)$ & $65(2)$ & $83(2)$ & $4(2)$ & $-8(2)$ & $5(2)$ \\
$\mathrm{C}(8 \mathrm{~A} 2)$ & $66(2)$ & $69(2)$ & $85(2)$ & $11(1)$ & $-1(2)$ & $10(1)$ \\
$\mathrm{C}(9 \mathrm{~A} 2)$ & $79(2)$ & $61(2)$ & $91(2)$ & $6(2)$ & $3(2)$ & $-1(1)$ \\
$\mathrm{C}\left(1^{\prime} \mathrm{B}\right)$ & $163(5)$ & $184(5)$ & $133(4)$ & $-59(4)$ & $-21(4)$ & $38(4)$ \\
$\mathrm{C}(2 ' \mathrm{~B})$ & $101(3)$ & $145(3)$ & $107(3)$ & $-25(3)$ & $-4(2)$ & $24(3)$ \\
\hline
\end{tabular}


Table 6. Hydrogen coordinates ( $\left.\mathrm{x} 10^{4}\right)$ and isotropic displacement parameters $\left(\AA^{2} \mathrm{x} 10^{3}\right)$ for $8 \mathrm{a}$.

\begin{tabular}{|c|c|c|c|c|}
\hline & $\mathrm{x}$ & $\mathrm{y}$ & $\mathrm{z}$ & $\mathrm{U}(\mathrm{eq})$ \\
\hline $\mathrm{H}\left(1^{\prime} \mathrm{A}\right)$ & 2586 & 8750 & -1860 & 143 \\
\hline $\mathrm{H}\left(3^{\prime} \mathrm{A}\right)$ & 3705 & 7948 & 7130 & 188 \\
\hline $\mathrm{H}\left(1^{\prime} \mathrm{F}\right)$ & 7836 & 7740 & 2942 & 173 \\
\hline $\mathrm{H}\left(1^{\prime} \mathrm{G}\right)$ & 6572 & 7222 & 2826 & 203 \\
\hline $\mathrm{H}(3 \mathrm{~A})$ & 5946 & 7078 & 2448 & 205 \\
\hline $\mathrm{H}(1 \mathrm{~A})$ & 2050 & 7073 & 1932 & 152 \\
\hline $\mathrm{H}(1 \mathrm{~B})$ & 2633 & 8293 & 2093 & 152 \\
\hline $\mathrm{H}(2 \mathrm{~A})$ & 3573 & 6109 & 2350 & 154 \\
\hline $\mathrm{H}(2 \mathrm{~B})$ & 4050 & 7321 & 2576 & 154 \\
\hline $\mathrm{H}(3 \mathrm{~B})$ & 4902 & 6092 & 1653 & 128 \\
\hline $\mathrm{H}(4 \mathrm{~A})$ & 6217 & 7855 & 936 & 97 \\
\hline $\mathrm{H}(4 \mathrm{~B})$ & 5258 & 6893 & 768 & 97 \\
\hline $\mathrm{H}(5 \mathrm{~A})$ & 6296 & 8425 & -70 & 98 \\
\hline $\mathrm{H}(5 \mathrm{~B})$ & 5261 & 7519 & -214 & 98 \\
\hline $\mathrm{H}(6 \mathrm{~A})$ & 5278 & 8947 & -878 & 101 \\
\hline $\mathrm{H}(6 \mathrm{~B})$ & 5172 & 9940 & -439 & 101 \\
\hline $\mathrm{H}(7 \mathrm{~A})$ & 3302 & 8338 & -711 & 85 \\
\hline $\mathrm{H}(8 \mathrm{~A})$ & 3092 & 10066 & 108 & 91 \\
\hline $\mathrm{H}(8 \mathrm{~B})$ & 2059 & 9169 & -50 & 91 \\
\hline $\mathrm{H}(9 \mathrm{~A})$ & 2853 & 9314 & 1095 & 104 \\
\hline $\mathrm{H}(9 \mathrm{~B})$ & 1899 & 8359 & 908 & 104 \\
\hline $\mathrm{H}(4 \mathrm{AA})$ & 5026 & 9313 & 572 & 86 \\
\hline $\mathrm{H}(8 \mathrm{AA})$ & 3247 & 7640 & 266 & 82 \\
\hline H(9AA) & 3232 & 6892 & 1180 & 106 \\
\hline $\mathrm{H}(3 \mathrm{AA})$ & 4859 & 9632 & 1433 & 168 \\
\hline $\mathrm{H}(3 \mathrm{AB})$ & 5864 & 9036 & 1799 & 168 \\
\hline $\mathrm{H}(3 \mathrm{AC})$ & 4505 & 9143 & 2009 & 168 \\
\hline $\mathrm{H}\left(2^{\prime} \mathrm{A}\right)$ & 2115 & 9879 & -995 & 104 \\
\hline $\mathrm{H}\left(2^{\prime} \mathrm{B}\right)$ & 3145 & 10775 & -838 & 104 \\
\hline $\mathrm{H}\left(1^{\prime} \mathrm{B}\right)$ & 3081 & 10517 & -1778 & 110 \\
\hline
\end{tabular}




\begin{tabular}{|c|c|c|c|c|}
\hline $\mathrm{H}\left(1^{\prime} \mathrm{C}\right)$ & 4369 & 10121 & -1552 & 110 \\
\hline $\mathrm{H}\left(1^{\prime} \mathrm{D}\right)$ & 5862 & 11653 & 6680 & 138 \\
\hline $\mathrm{H}\left(1^{\prime} \mathrm{E}\right)$ & 6897 & 10715 & 6797 & 138 \\
\hline $\mathrm{H}\left(2^{\prime} \mathrm{C}\right)$ & 5712 & 9956 & 7444 & 120 \\
\hline $\mathrm{H}\left(2^{\prime} \mathrm{D}\right)$ & 4633 & 10821 & 7291 & 120 \\
\hline $\mathrm{H}\left(3^{\prime} \mathrm{B}\right)$ & 3754 & 9556 & 6721 & 106 \\
\hline $\mathrm{H}\left(4^{\prime} \mathrm{A}\right)$ & 3970 & 8651 & 5830 & 110 \\
\hline $\mathrm{H}\left(4^{\prime} \mathrm{B}\right)$ & 4622 & 7490 & 6011 & 110 \\
\hline $\mathrm{H}\left(5^{\prime} \mathrm{A}\right)$ & 4140 & 8140 & 4830 & 133 \\
\hline $\mathrm{H}\left(5^{\prime} \mathrm{B}\right)$ & 4692 & 6930 & 5007 & 133 \\
\hline $\mathrm{H}\left(6^{\prime} \mathrm{A}\right)$ & 6405 & 7215 & 4463 & 137 \\
\hline $\mathrm{H}\left(6^{\prime} \mathrm{B}\right)$ & 5226 & 7376 & 4102 & 137 \\
\hline $\mathrm{H}\left(7^{\prime} \mathrm{A}\right)$ & 5393 & 9353 & 4139 & 108 \\
\hline $\mathrm{H}\left(8^{\prime} \mathrm{A}\right)$ & 7490 & 9116 & 4809 & 107 \\
\hline $\mathrm{H}\left(8^{\prime} \mathrm{B}\right)$ & 6923 & 10310 & 4619 & 107 \\
\hline $\mathrm{H}\left(9^{\prime} \mathrm{A}\right)$ & 7315 & 9835 & 5789 & 102 \\
\hline $\mathrm{H}\left(9^{\prime} \mathrm{B}\right)$ & 6695 & 11006 & 5591 & 102 \\
\hline $\mathrm{H}(3 \mathrm{BA})$ & 6877 & 8696 & 6880 & 166 \\
\hline $\mathrm{H}(3 \mathrm{BB})$ & 7101 & 8200 & 6292 & 166 \\
\hline $\mathrm{H}(3 \mathrm{BC})$ & 6277 & 7504 & 6705 & 166 \\
\hline $\mathrm{H}(4 \mathrm{AB})$ & 6272 & 7860 & 5460 & 96 \\
\hline $\mathrm{H}(8 \mathrm{AB})$ & 5177 & 9977 & 5134 & 88 \\
\hline $\mathrm{H}(9 \mathrm{AB})$ & 4922 & 10534 & 6063 & 92 \\
\hline $\mathrm{H}(1 \mathrm{BB})$ & 6148 & 7656 & 3317 & 192 \\
\hline $\mathrm{H}(1 \mathrm{BA})$ & 5867 & 8926 & 3105 & 192 \\
\hline $\mathrm{H}(2 \mathrm{BA})$ & 7237 & 9711 & 3685 & 141 \\
\hline $\mathrm{H}(2 \mathrm{BB})$ & 7729 & 8474 & 3851 & 141 \\
\hline
\end{tabular}


Projection view of 8 a with $25 \%$ thermal ellipsoids.

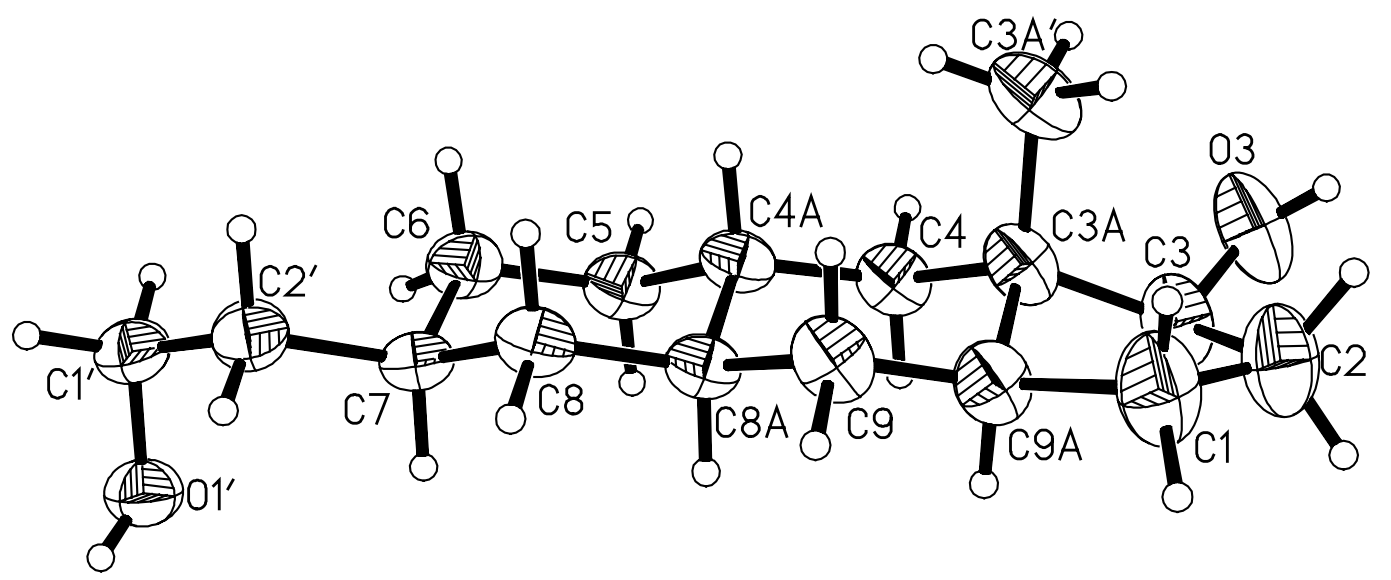


Table 7. Crystal data and structure refinement for $1 \mathrm{c}$.

Name of the Compound [3a $S, 4 \mathrm{a} R, 6 R, 8 \mathrm{a} R, 9 \mathrm{a} S]$-Dodecahydro-6-(2-hydroxyethyl)-9a-methyl-1 $H$ benz $[f]$ inden-1-one

Empirical formula

Formula weight

Temperature

Wavelength

Crystal system

Space group

Unit cell dimensions

Volume

Z

Density (calculated)

Absorption coefficient

$\mathrm{F}(000)$

Crystal size

Theta range for data collection

Index ranges

Reflections collected

Independent reflections

Completeness to theta $=29.00^{\circ}$

Absorption correction

Max. and min. transmission

Refinement method

Data / restraints / parameters

Goodness-of-fit on $\mathrm{F}^{2}$

Final $\mathrm{R}$ indices [I $>2 \operatorname{sigma}(\mathrm{I})]$

$\mathrm{R}$ indices (all data)

Absolute structure parameter

Largest diff. peak and hole
$\mathrm{C}_{16} \mathrm{H}_{26} \mathrm{O}_{2}$

250.37

170(2) K

$0.71073 \AA$

Orthorhombic

$\mathrm{P} 2{ }_{1} 2_{1} 2_{1}$

$\mathrm{a}=7.60850(10) \AA \quad \alpha=90^{\circ}$.

$\mathrm{b}=10.0606(2) \AA \quad \beta=90^{\circ}$.

$\mathrm{c}=18.9191(4) \AA \quad \gamma=90^{\circ}$.

$1448.18(5) \AA^{3}$

4

$1.148 \mathrm{Mg} / \mathrm{m}^{3}$

$0.073 \mathrm{~mm}^{-1}$

552

$0.41 \times 0.37 \times 0.24 \mathrm{~mm}^{3}$

2.15 to $29.00^{\circ}$.

$-10 \leq \mathrm{h} \leq 10,-13 \leq \mathrm{k} \leq 13,-25 \leq \mathrm{l} \leq 25$

19816

$3735[\mathrm{R}$ (int) $=0.026]$

$99.0 \%$

Semi-empirical from equivalents

0.9826 and 0.9706

Full-matrix least-squares on $\mathrm{F}^{2}$

3735 / 0 / 267

1.034

$\mathrm{R} 1=0.0376, \mathrm{wR} 2=0.0987$

$\mathrm{R} 1=0.0446, \mathrm{wR} 2=0.1033$

$0.1(11)$

0.235 and -0.148 e. $\AA^{-3}$ 
Table 8. Atomic coordinates ( $\mathrm{x} 10^{4}$ ) and equivalent isotropic displacement parameters $\left(\AA^{2} \times 10^{3}\right)$ for 1c. $U(e q)$ is defined as one third of the trace of the orthogonalized $U^{i j}$ tensor.

\begin{tabular}{lrrrr}
\hline & $\mathrm{x}$ & $\mathrm{y}$ & $\mathrm{z}$ & $\mathrm{U}(\mathrm{eq})$ \\
\hline $\mathrm{O}(1)$ & & & & \\
$\mathrm{O}\left(2^{\prime}\right)$ & $8171(1)$ & $13303(1)$ & $2221(1)$ & $47(1)$ \\
$\mathrm{C}(1)$ & $11596(2)$ & $4361(1)$ & $1957(1)$ & $58(1)$ \\
$\mathrm{C}(2)$ & $7031(2)$ & $12621(1)$ & $1961(1)$ & $37(1)$ \\
$\mathrm{C}(3)$ & $5158(2)$ & $12482(2)$ & $2245(1)$ & $53(1)$ \\
$\mathrm{C}(4)$ & $4238(2)$ & $11490(2)$ & $1760(1)$ & $51(1)$ \\
$\mathrm{C}(5)$ & $5523(2)$ & $9797(1)$ & $818(1)$ & $38(1)$ \\
$\mathrm{C}(6)$ & $7710(2)$ & $8009(1)$ & $1163(1)$ & $29(1)$ \\
$\mathrm{C}(7)$ & $9419(2)$ & $7273(1)$ & $996(1)$ & $29(1)$ \\
$\mathrm{C}(8)$ & $10920(2)$ & $8283(1)$ & $947(1)$ & $31(1)$ \\
$\mathrm{C}(9)$ & $10528(2)$ & $9367(1)$ & $403(1)$ & $33(1)$ \\
$\mathrm{C}(10)$ & $8958(2)$ & $11090(1)$ & $1167(1)$ & $29(1)$ \\
$\mathrm{C}(3 \mathrm{~A})$ & $6721(2)$ & $12748(2)$ & $697(1)$ & $42(1)$ \\
$\mathrm{C}(4 \mathrm{~A})$ & $5783(2)$ & $10712(1)$ & $1444(1)$ & $34(1)$ \\
$\mathrm{C}(8 \mathrm{~A})$ & $7273(2)$ & $9107(1)$ & $631(1)$ & $31(1)$ \\
$\mathrm{C}(9 \mathrm{~A})$ & $8800(2)$ & $10110(1)$ & $545(1)$ & $29(1)$ \\
$\mathrm{C}\left(1^{\prime}\right)$ & $7190(2)$ & $11775(1)$ & $1299(1)$ & $30(1)$ \\
$\mathrm{C}\left(2^{\prime}\right)$ & $9754(2)$ & $6202(1)$ & $1553(1)$ & $34(1)$ \\
& $11298(2)$ & $5287(2)$ & $1402(1)$ & $48(1)$ \\
& & & & \\
\hline
\end{tabular}


Table 9. Bond lengths $[\AA]$ and angles $\left[{ }^{\circ}\right]$ for $1 \mathrm{c}$.

\begin{tabular}{|c|c|}
\hline $\mathrm{O}(1)-\mathrm{C}(1)$ & $1.2099(17)$ \\
\hline $\mathrm{O}\left(2^{\prime}\right)-\mathrm{C}\left(2^{\prime}\right)$ & $1.4220(18)$ \\
\hline $\mathrm{O}\left(2^{\prime}\right)-\mathrm{H}\left(2^{\prime}\right)$ & $0.90(2)$ \\
\hline $\mathrm{C}(1)-\mathrm{C}(9 \mathrm{~A})$ & $1.5186(17)$ \\
\hline $\mathrm{C}(1)-\mathrm{C}(2)$ & $1.529(2)$ \\
\hline$C(2)-C(3)$ & $1.526(3)$ \\
\hline $\mathrm{C}(2)-\mathrm{H}(2 \mathrm{~A})$ & $1.02(2)$ \\
\hline $\mathrm{C}(2)-\mathrm{H}(2 \mathrm{~B})$ & $0.96(2)$ \\
\hline $\mathrm{C}(3)-\mathrm{C}(3 \mathrm{~A})$ & $1.5341(17)$ \\
\hline $\mathrm{C}(3)-\mathrm{H}(3 \mathrm{~A})$ & $0.98(2)$ \\
\hline $\mathrm{C}(3)-\mathrm{H}(3 \mathrm{~B})$ & $0.97(2)$ \\
\hline $\mathrm{C}(4)-\mathrm{C}(3 \mathrm{~A})$ & $1.5120(19)$ \\
\hline $\mathrm{C}(4)-\mathrm{C}(4 \mathrm{~A})$ & $1.5435(17)$ \\
\hline $\mathrm{C}(4)-\mathrm{H}(4 \mathrm{~A})$ & $0.951(17)$ \\
\hline $\mathrm{C}(4)-\mathrm{H}(4 \mathrm{~B})$ & $0.998(18)$ \\
\hline$C(5)-C(6)$ & $1.5296(15)$ \\
\hline $\mathrm{C}(5)-\mathrm{C}(4 \mathrm{~A})$ & $1.5319(16)$ \\
\hline $\mathrm{C}(5)-\mathrm{H}(5 \mathrm{~A})$ & $0.970(13)$ \\
\hline $\mathrm{C}(5)-\mathrm{H}(5 \mathrm{~B})$ & $0.937(16)$ \\
\hline$C(6)-C\left(1^{\prime}\right)$ & $1.5288(17)$ \\
\hline$C(6)-C(7)$ & $1.5317(16)$ \\
\hline $\mathrm{C}(6)-\mathrm{H}(6)$ & $1.017(15)$ \\
\hline$C(7)-C(8)$ & $1.5284(17)$ \\
\hline $\mathrm{C}(7)-\mathrm{H}(7 \mathrm{~A})$ & $0.970(17)$ \\
\hline $\mathrm{C}(7)-\mathrm{H}(7 \mathrm{~B})$ & $0.989(14)$ \\
\hline $\mathrm{C}(8)-\mathrm{C}(8 \mathrm{~A})$ & $1.5364(16)$ \\
\hline $\mathrm{C}(8)-\mathrm{H}(8 \mathrm{~A})$ & $0.980(16)$ \\
\hline $\mathrm{C}(8)-\mathrm{H}(8 \mathrm{~B})$ & $0.988(16)$ \\
\hline $\mathrm{C}(9)-\mathrm{C}(9 \mathrm{~A})$ & $1.5322(15)$ \\
\hline $\mathrm{C}(9)-\mathrm{C}(8 \mathrm{~A})$ & $1.5407(16)$ \\
\hline $\mathrm{C}(9)-\mathrm{H}(9 \mathrm{~A})$ & $0.983(14)$ \\
\hline $\mathrm{C}(9)-\mathrm{H}(9 \mathrm{~B})$ & $0.980(14)$ \\
\hline $\mathrm{C}(10)-\mathrm{C}(9 \mathrm{~A})$ & $1.5443(17)$ \\
\hline $\mathrm{C}(10)-\mathrm{H}(10 \mathrm{~A})$ & $0.98(2)$ \\
\hline
\end{tabular}




\begin{tabular}{|c|c|}
\hline $\mathrm{C}(10)-\mathrm{H}(10 \mathrm{~B})$ & $0.946(18)$ \\
\hline $\mathrm{C}(10)-\mathrm{H}(10 \mathrm{C})$ & $0.94(2)$ \\
\hline$C(3 A)-C(9 A)$ & $1.5377(17)$ \\
\hline $\mathrm{C}(3 \mathrm{~A})-\mathrm{H}(3)$ & $0.996(15)$ \\
\hline $\mathrm{C}(4 \mathrm{~A})-\mathrm{C}(8 \mathrm{~A})$ & $1.5473(16)$ \\
\hline $\mathrm{C}(4 \mathrm{~A})-\mathrm{H}(4)$ & $1.009(15)$ \\
\hline $\mathrm{C}(8 \mathrm{~A})-\mathrm{H}(8)$ & $1.005(16)$ \\
\hline $\mathrm{C}\left(1^{\prime}\right)-\mathrm{C}\left(2^{\prime}\right)$ & $1.5195(18)$ \\
\hline $\mathrm{C}\left(1^{\prime}\right)-\mathrm{H}\left(1^{\prime} \mathrm{A}\right)$ & $0.991(18)$ \\
\hline C(1')-H(1'B) & $1.018(18)$ \\
\hline $\mathrm{C}\left(2^{\prime}\right)-\mathrm{H}\left(2^{\prime} \mathrm{A}\right)$ & $1.03(2)$ \\
\hline $\mathrm{C}\left(2^{\prime}\right)-\mathrm{H}\left(2^{\prime} \mathrm{B}\right)$ & $0.94(2)$ \\
\hline $\mathrm{C}\left(2^{\prime}\right)-\mathrm{O}\left(2^{\prime}\right)-\mathrm{H}\left(2^{\prime}\right)$ & $108.0(13)$ \\
\hline $\mathrm{O}(1)-\mathrm{C}(1)-\mathrm{C}(9 \mathrm{~A})$ & $126.51(12)$ \\
\hline $\mathrm{O}(1)-\mathrm{C}(1)-\mathrm{C}(2)$ & $125.26(13)$ \\
\hline $\mathrm{C}(9 \mathrm{~A})-\mathrm{C}(1)-\mathrm{C}(2)$ & $108.22(12)$ \\
\hline $\mathrm{C}(3)-\mathrm{C}(2)-\mathrm{C}(1)$ & $106.04(12)$ \\
\hline $\mathrm{C}(3)-\mathrm{C}(2)-\mathrm{H}(2 \mathrm{~A})$ & $110.0(11)$ \\
\hline $\mathrm{C}(1)-\mathrm{C}(2)-\mathrm{H}(2 \mathrm{~A})$ & $107.9(11)$ \\
\hline $\mathrm{C}(3)-\mathrm{C}(2)-\mathrm{H}(2 \mathrm{~B})$ & 113.3(14) \\
\hline $\mathrm{C}(1)-\mathrm{C}(2)-\mathrm{H}(2 \mathrm{~B})$ & $113.1(14)$ \\
\hline $\mathrm{H}(2 \mathrm{~A})-\mathrm{C}(2)-\mathrm{H}(2 \mathrm{~B})$ & $106.5(18)$ \\
\hline $\mathrm{C}(2)-\mathrm{C}(3)-\mathrm{C}(3 \mathrm{~A})$ & $102.51(12)$ \\
\hline $\mathrm{C}(2)-\mathrm{C}(3)-\mathrm{H}(3 \mathrm{~A})$ & $109.6(12)$ \\
\hline $\mathrm{C}(3 \mathrm{~A})-\mathrm{C}(3)-\mathrm{H}(3 \mathrm{~A})$ & $111.5(12)$ \\
\hline $\mathrm{C}(2)-\mathrm{C}(3)-\mathrm{H}(3 \mathrm{~B})$ & $114.9(12)$ \\
\hline $\mathrm{C}(3 \mathrm{~A})-\mathrm{C}(3)-\mathrm{H}(3 \mathrm{~B})$ & $113.4(13)$ \\
\hline $\mathrm{H}(3 \mathrm{~A})-\mathrm{C}(3)-\mathrm{H}(3 \mathrm{~B})$ & $105.1(17)$ \\
\hline$C(3 A)-C(4)-C(4 A)$ & 109.93(10) \\
\hline $\mathrm{C}(3 \mathrm{~A})-\mathrm{C}(4)-\mathrm{H}(4 \mathrm{~A})$ & $114.2(10)$ \\
\hline $\mathrm{C}(4 \mathrm{~A})-\mathrm{C}(4)-\mathrm{H}(4 \mathrm{~A})$ & $106.6(10)$ \\
\hline $\mathrm{C}(3 \mathrm{~A})-\mathrm{C}(4)-\mathrm{H}(4 \mathrm{~B})$ & $111.8(10)$ \\
\hline $\mathrm{C}(4 \mathrm{~A})-\mathrm{C}(4)-\mathrm{H}(4 \mathrm{~B})$ & $109.6(10)$ \\
\hline $\mathrm{H}(4 \mathrm{~A})-\mathrm{C}(4)-\mathrm{H}(4 \mathrm{~B})$ & $104.4(14)$ \\
\hline $\mathrm{C}(6)-\mathrm{C}(5)-\mathrm{C}(4 \mathrm{~A})$ & $113.42(10)$ \\
\hline
\end{tabular}




\begin{tabular}{|c|c|}
\hline $\mathrm{C}(6)-\mathrm{C}(5)-\mathrm{H}(5 \mathrm{~A})$ & $107.6(9)$ \\
\hline $\mathrm{C}(4 \mathrm{~A})-\mathrm{C}(5)-\mathrm{H}(5 \mathrm{~A})$ & $111.6(8)$ \\
\hline $\mathrm{C}(6)-\mathrm{C}(5)-\mathrm{H}(5 \mathrm{~B})$ & $109.2(9)$ \\
\hline $\mathrm{C}(4 \mathrm{~A})-\mathrm{C}(5)-\mathrm{H}(5 \mathrm{~B})$ & 107.1(9) \\
\hline $\mathrm{H}(5 \mathrm{~A})-\mathrm{C}(5)-\mathrm{H}(5 \mathrm{~B})$ & $107.8(12)$ \\
\hline$C\left(1^{\prime}\right)-C(6)-C(5)$ & $109.88(10)$ \\
\hline $\mathrm{C}\left(1^{\prime}\right)-\mathrm{C}(6)-\mathrm{C}(7)$ & $112.69(10)$ \\
\hline$C(5)-C(6)-C(7)$ & $108.98(9)$ \\
\hline $\mathrm{C}\left(1^{\prime}\right)-\mathrm{C}(6)-\mathrm{H}(6)$ & $107.9(9)$ \\
\hline $\mathrm{C}(5)-\mathrm{C}(6)-\mathrm{H}(6)$ & $109.0(9)$ \\
\hline $\mathrm{C}(7)-\mathrm{C}(6)-\mathrm{H}(6)$ & 108.3(9) \\
\hline $\mathrm{C}(8)-\mathrm{C}(7)-\mathrm{C}(6)$ & $111.68(10)$ \\
\hline $\mathrm{C}(8)-\mathrm{C}(7)-\mathrm{H}(7 \mathrm{~A})$ & 109.4(9) \\
\hline $\mathrm{C}(6)-\mathrm{C}(7)-\mathrm{H}(7 \mathrm{~A})$ & $110.0(9)$ \\
\hline $\mathrm{C}(8)-\mathrm{C}(7)-\mathrm{H}(7 \mathrm{~B})$ & $109.2(8)$ \\
\hline $\mathrm{C}(6)-\mathrm{C}(7)-\mathrm{H}(7 \mathrm{~B})$ & $106.0(9)$ \\
\hline $\mathrm{H}(7 \mathrm{~A})-\mathrm{C}(7)-\mathrm{H}(7 \mathrm{~B})$ & $110.5(13)$ \\
\hline $\mathrm{C}(7)-\mathrm{C}(8)-\mathrm{C}(8 \mathrm{~A})$ & $113.34(9)$ \\
\hline $\mathrm{C}(7)-\mathrm{C}(8)-\mathrm{H}(8 \mathrm{~A})$ & $111.0(10)$ \\
\hline $\mathrm{C}(8 \mathrm{~A})-\mathrm{C}(8)-\mathrm{H}(8 \mathrm{~A})$ & $106.5(9)$ \\
\hline $\mathrm{C}(7)-\mathrm{C}(8)-\mathrm{H}(8 \mathrm{~B})$ & $110.1(9)$ \\
\hline $\mathrm{C}(8 \mathrm{~A})-\mathrm{C}(8)-\mathrm{H}(8 \mathrm{~B})$ & 108.3(9) \\
\hline $\mathrm{H}(8 \mathrm{~A})-\mathrm{C}(8)-\mathrm{H}(8 \mathrm{~B})$ & $107.3(12)$ \\
\hline $\mathrm{C}(9 \mathrm{~A})-\mathrm{C}(9)-\mathrm{C}(8 \mathrm{~A})$ & $110.13(9)$ \\
\hline $\mathrm{C}(9 \mathrm{~A})-\mathrm{C}(9)-\mathrm{H}(9 \mathrm{~A})$ & 109.3(9) \\
\hline $\mathrm{C}(8 \mathrm{~A})-\mathrm{C}(9)-\mathrm{H}(9 \mathrm{~A})$ & $110.5(8)$ \\
\hline $\mathrm{C}(9 \mathrm{~A})-\mathrm{C}(9)-\mathrm{H}(9 \mathrm{~B})$ & $110.9(8)$ \\
\hline $\mathrm{C}(8 \mathrm{~A})-\mathrm{C}(9)-\mathrm{H}(9 \mathrm{~B})$ & 107.2(8) \\
\hline $\mathrm{H}(9 \mathrm{~A})-\mathrm{C}(9)-\mathrm{H}(9 \mathrm{~B})$ & $108.8(12)$ \\
\hline $\mathrm{C}(9 \mathrm{~A})-\mathrm{C}(10)-\mathrm{H}(10 \mathrm{~A})$ & $109.9(11)$ \\
\hline $\mathrm{C}(9 \mathrm{~A})-\mathrm{C}(10)-\mathrm{H}(10 \mathrm{~B})$ & $111.3(10)$ \\
\hline $\mathrm{H}(10 \mathrm{~A})-\mathrm{C}(10)-\mathrm{H}(10 \mathrm{~B})$ & $110.6(14)$ \\
\hline $\mathrm{C}(9 \mathrm{~A})-\mathrm{C}(10)-\mathrm{H}(10 \mathrm{C})$ & $109.5(12)$ \\
\hline $\mathrm{H}(10 \mathrm{~A})-\mathrm{C}(10)-\mathrm{H}(10 \mathrm{C})$ & $105.8(16)$ \\
\hline $\mathrm{H}(10 \mathrm{~B})-\mathrm{C}(10)-\mathrm{H}(10 \mathrm{C})$ & $109.5(14)$ \\
\hline $\mathrm{C}(4)-\mathrm{C}(3 \mathrm{~A})-\mathrm{C}(3)$ & $121.03(11)$ \\
\hline
\end{tabular}




\begin{tabular}{|c|c|}
\hline $\mathrm{C}(4)-\mathrm{C}(3 \mathrm{~A})-\mathrm{C}(9 \mathrm{~A})$ & $112.07(10)$ \\
\hline $\mathrm{C}(3)-\mathrm{C}(3 \mathrm{~A})-\mathrm{C}(9 \mathrm{~A})$ & $104.34(11)$ \\
\hline $\mathrm{C}(4)-\mathrm{C}(3 \mathrm{~A})-\mathrm{H}(3)$ & $106.9(9)$ \\
\hline $\mathrm{C}(3)-\mathrm{C}(3 \mathrm{~A})-\mathrm{H}(3)$ & 107.2(9) \\
\hline $\mathrm{C}(9 \mathrm{~A})-\mathrm{C}(3 \mathrm{~A})-\mathrm{H}(3)$ & 104.1(9) \\
\hline C(5)-C(4A)-C(4) & $111.14(10)$ \\
\hline$C(5)-C(4 A)-C(8 A)$ & $112.09(9)$ \\
\hline$C(4)-C(4 A)-C(8 A)$ & $112.22(10)$ \\
\hline $\mathrm{C}(5)-\mathrm{C}(4 \mathrm{~A})-\mathrm{H}(4)$ & 108.0(9) \\
\hline $\mathrm{C}(4)-\mathrm{C}(4 \mathrm{~A})-\mathrm{H}(4)$ & $107.7(9)$ \\
\hline $\mathrm{C}(8 \mathrm{~A})-\mathrm{C}(4 \mathrm{~A})-\mathrm{H}(4)$ & 105.3(9) \\
\hline $\mathrm{C}(8)-\mathrm{C}(8 \mathrm{~A})-\mathrm{C}(9)$ & $112.22(9)$ \\
\hline $\mathrm{C}(8)-\mathrm{C}(8 \mathrm{~A})-\mathrm{C}(4 \mathrm{~A})$ & $110.12(9)$ \\
\hline $\mathrm{C}(9)-\mathrm{C}(8 \mathrm{~A})-\mathrm{C}(4 \mathrm{~A})$ & $113.35(9)$ \\
\hline $\mathrm{C}(8)-\mathrm{C}(8 \mathrm{~A})-\mathrm{H}(8)$ & $109.5(9)$ \\
\hline $\mathrm{C}(9)-\mathrm{C}(8 \mathrm{~A})-\mathrm{H}(8)$ & 106.1(9) \\
\hline $\mathrm{C}(4 \mathrm{~A})-\mathrm{C}(8 \mathrm{~A})-\mathrm{H}(8)$ & $105.1(9)$ \\
\hline $\mathrm{C}(1)-\mathrm{C}(9 \mathrm{~A})-\mathrm{C}(9)$ & $117.15(10)$ \\
\hline$C(1)-C(9 A)-C(3 A)$ & $100.81(10)$ \\
\hline $\mathrm{C}(9)-\mathrm{C}(9 \mathrm{~A})-\mathrm{C}(3 \mathrm{~A})$ & 109.09(9) \\
\hline C(1)-C(9A)-C(10) & $103.66(10)$ \\
\hline $\mathrm{C}(9)-\mathrm{C}(9 \mathrm{~A})-\mathrm{C}(10)$ & $111.54(10)$ \\
\hline $\mathrm{C}(3 \mathrm{~A})-\mathrm{C}(9 \mathrm{~A})-\mathrm{C}(10)$ & $114.29(11)$ \\
\hline $\mathrm{C}\left(2^{\prime}\right)-\mathrm{C}\left(1^{\prime}\right)-\mathrm{C}(6)$ & $115.17(11)$ \\
\hline $\mathrm{C}\left(2^{\prime}\right)-\mathrm{C}\left(1^{\prime}\right)-\mathrm{H}\left(1^{\prime} \mathrm{A}\right)$ & 106.4(9) \\
\hline $\mathrm{C}(6)-\mathrm{C}\left(1^{\prime}\right)-\mathrm{H}\left(1^{\prime} \mathrm{A}\right)$ & $110.1(9)$ \\
\hline $\mathrm{C}\left(2^{\prime}\right)-\mathrm{C}\left(1^{\prime}\right)-\mathrm{H}\left(1^{\prime} \mathrm{B}\right)$ & $110.9(10)$ \\
\hline $\mathrm{C}(6)-\mathrm{C}\left(1^{\prime}\right)-\mathrm{H}\left(1^{\prime} \mathrm{B}\right)$ & $108.0(10)$ \\
\hline $\mathrm{H}\left(1^{\prime} \mathrm{A}\right)-\mathrm{C}\left(1^{\prime}\right)-\mathrm{H}\left(1^{\prime} \mathrm{B}\right)$ & $105.9(13)$ \\
\hline $\mathrm{O}\left(2^{\prime}\right)-\mathrm{C}\left(2^{\prime}\right)-\mathrm{C}\left(1^{\prime}\right)$ & $112.40(12)$ \\
\hline $\mathrm{O}\left(2^{\prime}\right)-\mathrm{C}\left(2^{\prime}\right)-\mathrm{H}\left(2^{\prime} \mathrm{A}\right)$ & $108.0(11)$ \\
\hline $\mathrm{C}\left(1^{\prime}\right)-\mathrm{C}\left(2^{\prime}\right)-\mathrm{H}\left(2^{\prime} \mathrm{A}\right)$ & $109.3(12)$ \\
\hline $\mathrm{O}\left(2^{\prime}\right)-\mathrm{C}\left(2^{\prime}\right)-\mathrm{H}\left(2^{\prime} \mathrm{B}\right)$ & $107.7(11)$ \\
\hline $\mathrm{C}\left(1^{\prime}\right)-\mathrm{C}\left(2^{\prime}\right)-\mathrm{H}\left(2^{\prime} \mathrm{B}\right)$ & $109.4(11)$ \\
\hline $\mathrm{H}\left(2^{\prime} \mathrm{A}\right)-\mathrm{C}\left(2^{\prime}\right)-\mathrm{H}\left(2^{\prime} \mathrm{B}\right)$ & $109.9(15)$ \\
\hline
\end{tabular}


Table 10. Anisotropic displacement parameters $\left(\AA^{2} \times 10^{3}\right)$ for $1 \mathrm{c}$. The anisotropic displacement factor exponent takes the form: $-2 \pi^{2}\left[h^{2} a^{* 2} U^{11}+\ldots+2 h k a^{*} b^{*} U^{12}\right]$

\begin{tabular}{lcccccc}
\hline & $\mathrm{U}^{11}$ & $\mathrm{U}^{22}$ & $\mathrm{U}^{33}$ & $\mathrm{U}^{23}$ & $\mathrm{U}^{13}$ & $\mathrm{U}^{12}$ \\
\hline $\mathrm{O}(1)$ & $60(1)$ & $37(1)$ & $44(1)$ & $-3(1)$ & $-6(1)$ & $2(1)$ \\
$\mathrm{O}\left(2^{\prime}\right)$ & $50(1)$ & $54(1)$ & $71(1)$ & $18(1)$ & $-10(1)$ & $12(1)$ \\
$\mathrm{C}(1)$ & $45(1)$ & $29(1)$ & $37(1)$ & $2(1)$ & $-1(1)$ & $10(1)$ \\
$\mathrm{C}(2)$ & $49(1)$ & $56(1)$ & $54(1)$ & $-6(1)$ & $12(1)$ & $17(1)$ \\
$\mathrm{C}(3)$ & $32(1)$ & $53(1)$ & $68(1)$ & $-3(1)$ & $12(1)$ & $8(1)$ \\
$\mathrm{C}(4)$ & $23(1)$ & $40(1)$ & $50(1)$ & $0(1)$ & $-5(1)$ & $0(1)$ \\
$\mathrm{C}(5)$ & $24(1)$ & $29(1)$ & $34(1)$ & $0(1)$ & $2(1)$ & $-3(1)$ \\
$\mathrm{C}(6)$ & $29(1)$ & $28(1)$ & $31(1)$ & $-3(1)$ & $1(1)$ & $-1(1)$ \\
$\mathrm{C}(7)$ & $25(1)$ & $32(1)$ & $36(1)$ & $-1(1)$ & $3(1)$ & $-1(1)$ \\
$\mathrm{C}(8)$ & $30(1)$ & $37(1)$ & $33(1)$ & $2(1)$ & $9(1)$ & $0(1)$ \\
$\mathrm{C}(9)$ & $26(1)$ & $28(1)$ & $32(1)$ & $3(1)$ & $0(1)$ & $-1(1)$ \\
$\mathrm{C}(10)$ & $49(1)$ & $39(1)$ & $40(1)$ & $9(1)$ & $-4(1)$ & $8(1)$ \\
$\mathrm{C}(3 \mathrm{~A})$ & $24(1)$ & $36(1)$ & $43(1)$ & $2(1)$ & $5(1)$ & $4(1)$ \\
$\mathrm{C}(4 \mathrm{~A})$ & $26(1)$ & $35(1)$ & $31(1)$ & $-3(1)$ & $-4(1)$ & $-1(1)$ \\
$\mathrm{C}(8 \mathrm{~A})$ & $28(1)$ & $33(1)$ & $26(1)$ & $2(1)$ & $1(1)$ & $0(1)$ \\
$\mathrm{C}(9 \mathrm{~A})$ & $31(1)$ & $29(1)$ & $31(1)$ & $3(1)$ & $-1(1)$ & $4(1)$ \\
$\mathrm{C}\left(1^{\prime}\right)$ & $36(1)$ & $30(1)$ & $38(1)$ & $0(1)$ & $1(1)$ & $1(1)$ \\
$\mathrm{C}\left(2^{\prime}\right)$ & $41(1)$ & $43(1)$ & $59(1)$ & $10(1)$ & $3(1)$ & $10(1)$ \\
\hline & & & & & & \\
\hline
\end{tabular}


Table 11. Hydrogen coordinates ( $\mathrm{x} 10^{4}$ ) and isotropic displacement parameters $\left(\AA^{2} \mathrm{x} 10^{3}\right)$ for $1 \mathrm{c}$.

\begin{tabular}{|c|c|c|c|c|}
\hline & $\mathrm{x}$ & $\mathrm{y}$ & $\mathrm{z}$ & $\mathrm{U}(\mathrm{eq})$ \\
\hline $\mathrm{H}\left(1^{\prime} \mathrm{A}\right)$ & $8710(20)$ & $5622(17)$ & 1601(8) & $46(4)$ \\
\hline $\mathrm{H}(1$ 'B) & $9900(20)$ & $6660(18)$ & 2030(10) & $55(5)$ \\
\hline $\mathrm{H}\left(2^{\prime}\right)$ & $10570(30)$ & $3950(20)$ & $2050(11)$ & $67(6)$ \\
\hline $\mathrm{H}\left(2^{\prime} \mathrm{A}\right)$ & $11050(30)$ & $4760(20)$ & $947(10)$ & $65(5)$ \\
\hline $\mathrm{H}\left(2^{\prime} \mathrm{B}\right)$ & $12320(30)$ & $5794(18)$ & 1348(9) & $53(5)$ \\
\hline $\mathrm{H}(2 \mathrm{~A})$ & $5230(20)$ & $12110(20)$ & $2745(11)$ & $70(6)$ \\
\hline $\mathrm{H}(2 \mathrm{~B})$ & $4560(30)$ & $13320(20)$ & $2281(12)$ & $83(7)$ \\
\hline $\mathrm{H}(3)$ & $6300(20)$ & $10165(16)$ & 1831(8) & $37(4)$ \\
\hline $\mathrm{H}(3 \mathrm{~A})$ & $3590(30)$ & $11970(20)$ & 1396(10) & $64(5)$ \\
\hline $\mathrm{H}(3 \mathrm{~B})$ & $3380(30)$ & $10930(20)$ & 1990(11) & $73(6)$ \\
\hline $\mathrm{H}(4)$ & $7130(20)$ & $8681(15)$ & $151(8)$ & $38(4)$ \\
\hline $\mathrm{H}(4 \mathrm{~A})$ & $4690(20)$ & $9110(17)$ & $895(8)$ & $46(4)$ \\
\hline $\mathrm{H}(4 \mathrm{~B})$ & $5070(20)$ & $10285(18)$ & $397(9)$ & $52(5)$ \\
\hline $\mathrm{H}(5 \mathrm{~A})$ & 7808(19) & $8357(14)$ & 1640(7) & $27(3)$ \\
\hline $\mathrm{H}(5 \mathrm{~B})$ & $6770(20)$ & $7404(15)$ & 1157(8) & $36(4)$ \\
\hline $\mathrm{H}(6)$ & $9300(20)$ & $6814(16)$ & $519(7)$ & $37(4)$ \\
\hline $\mathrm{H}(7 \mathrm{~A})$ & $12000(20)$ & $7835(15)$ & $821(8)$ & $42(4)$ \\
\hline $\mathrm{H}(7 \mathrm{~B})$ & $11020(20)$ & $8691(14)$ & 1420(7) & $31(4)$ \\
\hline $\mathrm{H}(8)$ & $8480(20)$ & $10659(16)$ & $120(8)$ & $40(4)$ \\
\hline $\mathrm{H}(8 \mathrm{~A})$ & $10430(20)$ & $8991(16)$ & $-73(9)$ & $44(4)$ \\
\hline $\mathrm{H}(8 \mathrm{~B})$ & $11490(20)$ & $10023(16)$ & $394(8)$ & $39(4)$ \\
\hline $\mathrm{H}(9 \mathrm{~A})$ & $9330(20)$ & $10623(14)$ & 1599(7) & $31(3)$ \\
\hline $\mathrm{H}(9 \mathrm{~B})$ & $9863(18)$ & $11740(14)$ & 1040(7) & $27(3)$ \\
\hline $\mathrm{H}(10 \mathrm{~A})$ & $7650(30)$ & $13412(19)$ & $645(10)$ & $60(5)$ \\
\hline $\mathrm{H}(10 \mathrm{~B})$ & $6540(20)$ & $12292(17)$ & 265(9) & $49(4)$ \\
\hline $\mathrm{H}(10 \mathrm{C})$ & $5700(30)$ & $13220(20)$ & $817(9)$ & $59(5)$ \\
\hline
\end{tabular}


Table 12. Torsion angles $\left[{ }^{\circ}\right]$ for $1 \mathrm{c}$.

\begin{tabular}{|c|c|}
\hline $\mathrm{O}(1)-\mathrm{C}(1)-\mathrm{C}(2)-\mathrm{C}(3)$ & $-178.19(14)$ \\
\hline $\mathrm{C}(9 \mathrm{~A})-\mathrm{C}(1)-\mathrm{C}(2)-\mathrm{C}(3)$ & $2.62(16)$ \\
\hline $\mathrm{C}(1)-\mathrm{C}(2)-\mathrm{C}(3)-\mathrm{C}(3 \mathrm{~A})$ & $22.86(17)$ \\
\hline $\mathrm{C}(4 \mathrm{~A})-\mathrm{C}(5)-\mathrm{C}(6)-\mathrm{C}\left(1^{\prime}\right)$ & $179.85(10)$ \\
\hline $\mathrm{C}(4 \mathrm{~A})-\mathrm{C}(5)-\mathrm{C}(6)-\mathrm{C}(7)$ & $55.93(12)$ \\
\hline $\mathrm{C}\left(1^{\prime}\right)-\mathrm{C}(6)-\mathrm{C}(7)-\mathrm{C}(8)$ & $-178.19(10)$ \\
\hline$C(5)-C(6)-C(7)-C(8)$ & $-55.95(12)$ \\
\hline $\mathrm{C}(6)-\mathrm{C}(7)-\mathrm{C}(8)-\mathrm{C}(8 \mathrm{~A})$ & $56.37(13)$ \\
\hline $\mathrm{C}(4 \mathrm{~A})-\mathrm{C}(4)-\mathrm{C}(3 \mathrm{~A})-\mathrm{C}(3)$ & $-177.82(13)$ \\
\hline $\mathrm{C}(4 \mathrm{~A})-\mathrm{C}(4)-\mathrm{C}(3 \mathrm{~A})-\mathrm{C}(9 \mathrm{~A})$ & $58.43(13)$ \\
\hline $\mathrm{C}(2)-\mathrm{C}(3)-\mathrm{C}(3 \mathrm{~A})-\mathrm{C}(4)$ & $-167.62(13)$ \\
\hline $\mathrm{C}(2)-\mathrm{C}(3)-\mathrm{C}(3 \mathrm{~A})-\mathrm{C}(9 \mathrm{~A})$ & $-40.30(16)$ \\
\hline $\mathrm{C}(6)-\mathrm{C}(5)-\mathrm{C}(4 \mathrm{~A})-\mathrm{C}(4)$ & 179.02(9) \\
\hline$C(6)-C(5)-C(4 A)-C(8 A)$ & $-54.50(13)$ \\
\hline $\mathrm{C}(3 \mathrm{~A})-\mathrm{C}(4)-\mathrm{C}(4 \mathrm{~A})-\mathrm{C}(5)$ & $74.96(13)$ \\
\hline $\mathrm{C}(3 \mathrm{~A})-\mathrm{C}(4)-\mathrm{C}(4 \mathrm{~A})-\mathrm{C}(8 \mathrm{~A})$ & $-51.45(14)$ \\
\hline $\mathrm{C}(7)-\mathrm{C}(8)-\mathrm{C}(8 \mathrm{~A})-\mathrm{C}(9)$ & 74.84(13) \\
\hline $\mathrm{C}(7)-\mathrm{C}(8)-\mathrm{C}(8 \mathrm{~A})-\mathrm{C}(4 \mathrm{~A})$ & $-52.43(13)$ \\
\hline $\mathrm{C}(9 \mathrm{~A})-\mathrm{C}(9)-\mathrm{C}(8 \mathrm{~A})-\mathrm{C}(8)$ & $-178.48(9)$ \\
\hline $\mathrm{C}(9 \mathrm{~A})-\mathrm{C}(9)-\mathrm{C}(8 \mathrm{~A})-\mathrm{C}(4 \mathrm{~A})$ & $-52.96(12)$ \\
\hline $\mathrm{C}(5)-\mathrm{C}(4 \mathrm{~A})-\mathrm{C}(8 \mathrm{~A})-\mathrm{C}(8)$ & $50.72(13)$ \\
\hline $\mathrm{C}(4)-\mathrm{C}(4 \mathrm{~A})-\mathrm{C}(8 \mathrm{~A})-\mathrm{C}(8)$ & $176.62(10)$ \\
\hline $\mathrm{C}(5)-\mathrm{C}(4 \mathrm{~A})-\mathrm{C}(8 \mathrm{~A})-\mathrm{C}(9)$ & $-75.91(12)$ \\
\hline $\mathrm{C}(4)-\mathrm{C}(4 \mathrm{~A})-\mathrm{C}(8 \mathrm{~A})-\mathrm{C}(9)$ & $49.98(13)$ \\
\hline $\mathrm{O}(1)-\mathrm{C}(1)-\mathrm{C}(9 \mathrm{~A})-\mathrm{C}(9)$ & $35.88(18)$ \\
\hline $\mathrm{C}(2)-\mathrm{C}(1)-\mathrm{C}(9 \mathrm{~A})-\mathrm{C}(9)$ & $-144.96(12)$ \\
\hline $\mathrm{O}(1)-\mathrm{C}(1)-\mathrm{C}(9 \mathrm{~A})-\mathrm{C}(3 \mathrm{~A})$ & $154.05(13)$ \\
\hline $\mathrm{C}(2)-\mathrm{C}(1)-\mathrm{C}(9 \mathrm{~A})-\mathrm{C}(3 \mathrm{~A})$ & $-26.78(13)$ \\
\hline $\mathrm{O}(1)-\mathrm{C}(1)-\mathrm{C}(9 \mathrm{~A})-\mathrm{C}(10)$ & $-87.43(15)$ \\
\hline $\mathrm{C}(2)-\mathrm{C}(1)-\mathrm{C}(9 \mathrm{~A})-\mathrm{C}(10)$ & $91.74(13)$ \\
\hline $\mathrm{C}(8 \mathrm{~A})-\mathrm{C}(9)-\mathrm{C}(9 \mathrm{~A})-\mathrm{C}(1)$ & $171.11(10)$ \\
\hline $\mathrm{C}(8 \mathrm{~A})-\mathrm{C}(9)-\mathrm{C}(9 \mathrm{~A})-\mathrm{C}(3 \mathrm{~A})$ & $57.49(12)$ \\
\hline $\mathrm{C}(8 \mathrm{~A})-\mathrm{C}(9)-\mathrm{C}(9 \mathrm{~A})-\mathrm{C}(10)$ & $-69.70(13)$ \\
\hline $\mathrm{C}(4)-\mathrm{C}(3 \mathrm{~A})-\mathrm{C}(9 \mathrm{~A})-\mathrm{C}(1)$ & $173.96(10)$ \\
\hline
\end{tabular}




$\begin{array}{lc}\mathrm{C}(3)-\mathrm{C}(3 \mathrm{~A})-\mathrm{C}(9 \mathrm{~A})-\mathrm{C}(1) & 41.30(13) \\ \mathrm{C}(4)-\mathrm{C}(3 \mathrm{~A})-\mathrm{C}(9 \mathrm{~A})-\mathrm{C}(9) & -62.14(13) \\ \mathrm{C}(3)-\mathrm{C}(3 \mathrm{~A})-\mathrm{C}(9 \mathrm{~A})-\mathrm{C}(9) & 165.20(11) \\ \mathrm{C}(4)-\mathrm{C}(3 \mathrm{~A})-\mathrm{C}(9 \mathrm{~A})-\mathrm{C}(10) & 63.48(14) \\ \mathrm{C}(3)-\mathrm{C}(3 \mathrm{~A})-\mathrm{C}(9 \mathrm{~A})-\mathrm{C}(10) & -69.18(14) \\ \mathrm{C}(5)-\mathrm{C}(6)-\mathrm{C}\left(1^{\prime}\right)-\mathrm{C}\left(2^{\prime}\right) & 173.07(11) \\ \mathrm{C}(7)-\mathrm{C}(6)-\mathrm{C}\left(1^{\prime}\right)-\mathrm{C}\left(2^{\prime}\right) & -65.20(14) \\ \mathrm{C}(6)-\mathrm{C}\left(1^{\prime}\right)-\mathrm{C}\left(2^{\prime}\right)-\mathrm{O}\left(2^{\prime}\right) & 176.90(12)\end{array}$


Projection view of $1 \mathrm{c}$ with $50 \%$ thermal ellipsoids:

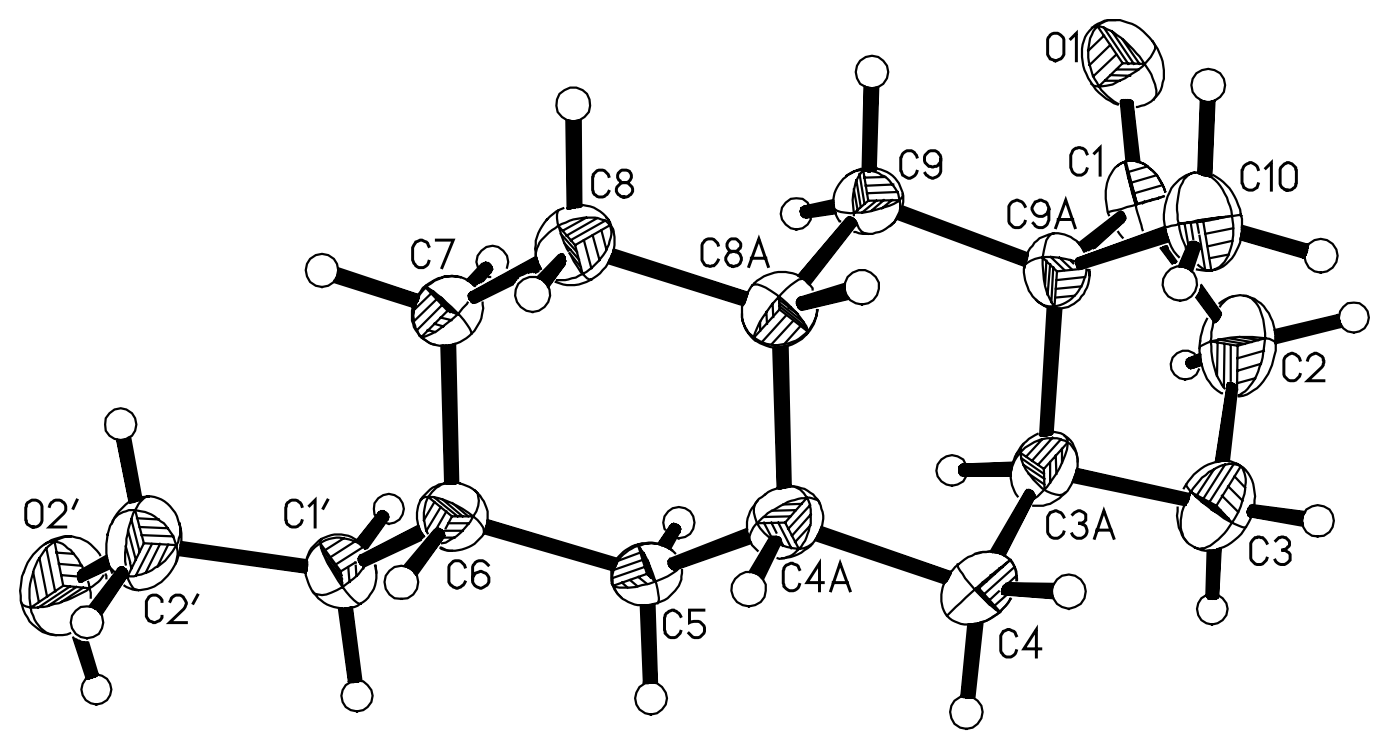

